\title{
Saturation of Depth and Polarization of Resonant and Satellite Spectral Holes Generated with Pulsed Laser Excitation
}

\author{
Wolfgang Drescher and Bernhard Dick* \\ Institut für Physikalische und Theoretische Chemie, Universität Regensburg, \\ 93053 Regensburg, Federal Republic of Germany
}

Received: October 26, 1993; In Final Form: January 4, $1994^{\circ}$

\begin{abstract}
We consider photochemical hole burning of an inhomogeneous and isotropic ensemble of chromophores through excitation with a narrow-bandwidth source of polarized light. We derive expressions for the depth and anisotropy of resonant and vibronic satellite spectral holes with increasing fluence. A fit of these saturation curves to experimental data yields the Debye-Waller factor of the system. A simple rate equation model predicts that the anisotropy of a spectral hole depends only on the angle between the transition dipoles of the burnt and the probed transitions. In experiments with pulsed lasers strong deviations from this ideal behavior are observed. An extended theory including saturation gives a satisfactory description of this phenomenon and provides a new method for the determination of photochemical quantum yields.
\end{abstract}

\section{Introduction}

A dilute solution of chromophores in a solid amorphous host such as a glass or polymer can be characterized by an inhomogeneous distribution of transition frequencies and an initially isotropic orientational distribution. Irradiation with a narrowbandwidth light source, e.g., a laser, excites only molecules which have their transition frequency within a homogeneous line width from the laser frequency. The excited subensemble is anisotropic since the probability of excitation depends on the angle $\beta$ between the light polarization vector and the transition dipole. A fraction $\Phi$ of these excited molecules is removed from the ensemble through a photochemical reaction, leaving a narrow hole in the inhomogeneous distribution function. The orientational distribution of the remaining molecules at the frequencies of this spectral hole is anisotropic, and the measured depth of the hole depends on the polarization of the probe light. The degree of polarization $\rho$ is frequently defined as

$$
\rho=\frac{H_{\|}-H_{\perp}}{H_{\|}+H_{\perp}}
$$

where $H_{\|}$and $H_{\perp}$ are the hole depths measured with the probe light polarized parallel or perpendicular to the burning light. A simple rate equation model predicts

$$
\rho=\frac{3 \cos ^{2} \delta-1}{\cos ^{2} \delta+3}
$$

in the limit of low burning fluence, where $\delta$ is the angle between the transition dipole moments of the burnt and the probed transitions. This equation is known as Perrin's formula ${ }^{1}$ in the context of fluorescence polarization. For resonant spectral holes burning and probing occurs on the same transition and the initial value of $\rho$ is $1 / 2$. Equation 2 suggests that for vibronic satellite holes $\rho$ might be useful for the determination of transition dipole moment directions and vibrational symmetries.

It is well-known that with increasing fluence the observed depth of the zero-phenon hole approaches a limiting value which is smaller than the initial absorbance of the sample. Apparently only a fraction of the initial absorbance can be burnt. This fraction may be identified with the contribution from the zero-phonon lines. Thus the asymptotic behavior of the hole depth should

- Author to whom correspondence should be addressed.

- Abstract published in Advance ACS Abstracts, February 15, 1994 yield the Debye-Waller factor of the system. This saturation is accompanied by an increase in the width of the spectral hole and a decrease in the anisotropy. We found that $\rho$ was already significantly smaller than $1 / 2$ for very shallow resonant spectral holes. When the sample was excited with laser pulses of moderate or high intensity the values for $\rho$ did not approach the value $1 / 2$ when extrapolated to zero hole depth.

Hence it is clear that a model theory is needed that describes the saturation behavior of $H_{\|}$and $\rho$ with sufficient accuracy to permit a fit to the experimental data. For the case of resonant spectral holes and low light intensities models of this kind have been treated by Osad'ko et al., ${ }^{2}$ Köhler et al., ${ }^{3}$ and Kador et al. ${ }^{4}$ In ref 3 the saturation of depth, width, and polarization degree of a resonant hole under the influence of continuous-wave (cw) excitation is simulated taking into account the possibility of a photochemical backreaction. Analytical formulas are given for the limits of small and very long burning times. In ref 4 the saturation behavior of the entire line-shape function of the zerophonon hole is discussed in detail. The Debye-Waller factor is obtained from a plot of the hole width against the hole depth. The initial slope of the hole width as a function of fluence gives the photochemical quantum yield.

Power effects in photochemical hole-burning are usually accounted for by considering the power broadening of the homogeneous line width of the resonantly excited transition.,3 In ref 3 a figure shows a simulation for the loss of anisotropy of a resonant hole with higher laser power. As far as we know, the saturation behavior of the depth and polarization of vibronic satellite holes has not yet been analyzed. In the following theoretical section we first find expressions for the anisotropic absorption and hole spectra in terms of the photochemically induced population change $\Delta N$ of the selectively excited subensemble and the joint distribution function of the two transitions involved in hole burning and probing. It is shown that part of the orientational average can be performed in a general way without prior knowledge of $\Delta N$. In the following step $\Delta N$ is calculated in the limits of weak (cw laser) excitation and intensive pulsed laser excitation. In the latter case the polarization degree does not approach Perrin's formula (eq 2) in the limit of small hole depth. The reason is that a single laser pulse excites a considerable fraction of the resonant subensemble. If all excited molecules would undergo photoreaction, this would lead to a deep hole with a correspondingly low anisotropy. Due to a small photochemical quantum yield, however, only a small fraction of this transient hole is permanently stored, albeit with the low 


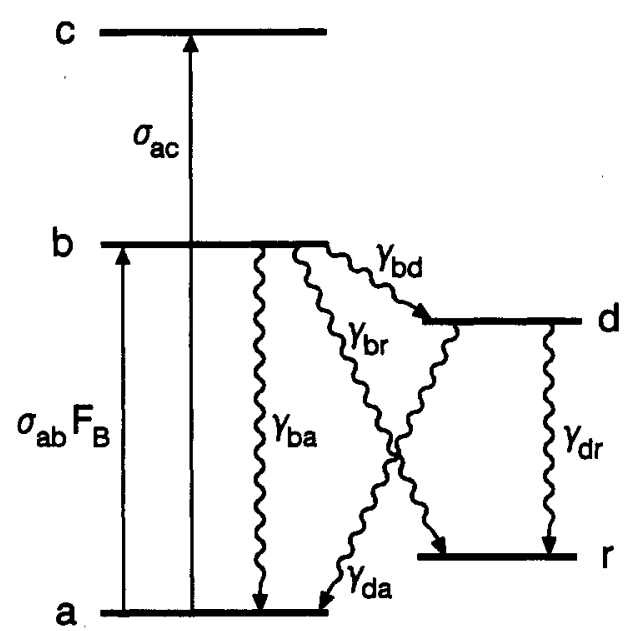

Figure 1. Level diagram for the molecular system considered. Spectral holes are burnt into the transition $a \rightarrow b$ (cross section $\sigma_{a b}$ ) and are detected on the transition $a \rightarrow c$ (cross section $\sigma_{a c}$ ). The various paths for decay are indicated by wavy lines labeled with the corresponding decay parameter.

anisotropy. The ratio of the observed permanent hole depth and the theoretical hole depth predicted for the observed initial anisotropy in the limit of low burning intensity is hence a measure of the photochemical quantum yield $\Phi$. This opens an independent route for the determination of $\Phi$ which does not depend on a precise knowledge of the laser intensity or the peak absorption cross section of the homogeneous lineshape.

This theoretical model is then applied to analyze the saturation behavior of depth and anisotropy observed with resonant and satellite spectral holes burnt with a pulsed dye laser into the absorption spectrum of tetraphenylporphine (TPP) in poly (methyl methacrylate) (PMMA). The results are compared to investigations on similar systems which analyze the broadening of resonant spectral holes with $\mathrm{cw}$-laser hole burning. Our method yields slightly lower values of the Debye-Waller factor and slightly higher values of the photochemical quantum yield. Possible reasons for these differences are discussed. In TPP the angle between transition moments is restricted to $0^{\circ}$ and $90^{\circ}$ by symmetry. A fit of the theoretical expression to the observed saturation of the polarization degree with increasing hole depth reproduces these angles with an accuracy of ca. $\pm 10^{\circ}$.

\section{Theory}

2.1. Anisotropic Distribution Functions and Hole Shape. We consider a molecule with a ground state $a$, two optically accessible states $b$ and $c$, a photoproduct state $r$, and a possible intermediate state $d$. Figure 1 shows the level structure and indicates the parameters considered for radiative and nonradiative transitions. The probability of finding a particular molecule in state $k$ is $N_{k}$, and all molecules are initially in the ground state, i.e., $N_{a}^{0}=1$. An individual molecule is characterized by the two optical transition frequencies $\omega_{b a}$ and $\omega_{c a}$ and by its orientation with respect to the laboratory frame, expressed as the set of Euler angles $\Omega=\{\alpha, \beta, \gamma\}$. The complete ensemble of molecules is described by a distribution function $\tilde{g}(\Omega, x, y)$, where $x=\omega_{b a}$ $\omega_{b a}^{0}$ and $y=\omega_{c a}-\omega_{c a}^{0}$ are the shifts of the transition frequencies from the center frequencies of the inhomogeneous distributions, $\omega_{b a}^{0}$ and $\omega_{c a}^{0}$. We assume that hole burning does not alter the parameters $\Omega, x$, and $y$ of an individual molecule and that the initial orientational distribution is isotropic and statistically independent of the spectral distribution:

$$
\tilde{g}_{0}(\Omega, x, y)=\left(1 / 8 \pi^{2}\right) g(x, y)
$$

The inhomogeneous distributions of $x$ and $y$ are in general not independent. Spectral hole burning is performed with light of frequency $\omega_{B}$ with polarization direction $e_{B}$ and photon flux $F_{B}$. The rate constant for the excitation of a particular subset of molecules is

$$
k_{X}=\sigma_{a b}\left(\omega_{B}-x\right)\left|\mathbf{e}_{B} \mathbf{e}_{a b}\right|^{2} F_{B}
$$

Here $\sigma_{a b}(\omega)$ is the homogeneous line-shape function of the molecules centered at $\omega_{b a}^{0}$, and $e_{a b}$ is a unit vector pointing into the direction of the molecular transition moment. We chose the $z$ axis of the molecular coordinate frame along this direction so that $\mathbf{e}_{B} \mathbf{e}_{a b}=\cos \beta$. After hole burning the population of the ground state $N_{a}$ will be a function of $x$ and $\cos \beta$. The new distribution function of the ensemble is then given by

$$
\tilde{g}(\Omega, x, y)=\left(1 / 8 \pi^{2}\right) g(x, y) N_{a}\left(\omega_{B}-x, \cos \beta\right)
$$

The particular form of the population $N_{a}\left(\omega_{B}-x, \cos \beta\right)$ depends on the model adopted for the hole-burning process. Several cases will be discussed below.

Following hole burning the ensemble is probed with light of polarization $e_{P}$ at frequency $\omega$ on the molecular transition $a \rightarrow$ c. If we denote the homogeneous line shape function of a molecule with the transition frequency $\omega_{c a}^{0}$ with $\sigma_{a c}(\omega)$, and the unit vector into the direction of the transition dipole with $\mathbf{e}_{a c}$, the absorption spectrum of the whole ensemble is given by

$$
A\left(\omega, \omega_{B}\right)=\int \mathrm{d} \Omega \int \mathrm{d} x \int \mathrm{d} y \tilde{g}(\Omega, x, y) \sigma_{a c}(\omega-y)\left|\mathbf{e}_{p} \mathbf{e}_{a d}\right|^{2}
$$

Before hole burning $\left(N_{a}=1\right)$ the absorption spectrum is

$$
A_{0}(\omega)=1 / 3 \int \mathrm{d} y g_{a c}(y) \sigma_{a c}(\omega-y)
$$

with the one-dimensional distribution function

$$
g_{a c}(y)=\int \mathrm{d} x g(x, y)
$$

When the two inhomogeneous distributions are statistically independent $\left(g(x, y)=g_{a b}(x) g_{a c}(y)\right)$, the spectrum after hole burning has the same shape as before hole burning, but with a polarization-dependent amplitude:

$$
\begin{gathered}
A\left(\omega, \omega_{B}\right)=A_{0}(\omega) X\left(\mathbf{e}_{B}, \mathbf{e}_{P}\right) \\
X\left(\mathbf{e}_{B}, \mathbf{e}_{P}\right)=3 \int \mathrm{d} \Omega / 8 \pi^{2} \int \mathrm{d} x g_{a b}(x) N_{a}\left(\omega_{B}-x, \cos \beta\right)\left|\mathrm{e}_{P} \mathbf{e}_{a c}\right|^{2}
\end{gathered}
$$

A narrow spectral hole may only be observed if the two inhomogeneous distributions are strongly correlated. This condition is very often fulfilled to a high degree if the two states $b$ and $c$ are vibrational substates of the same electronic state. A useful assumption for this case $^{6}$ is that the inhomogeneous distributions for the electronic transition $a \rightarrow b$ and the vibrational energy separation $b \rightarrow c$ are independent:

$$
g(x, y)=g_{e}(x) g_{v}(x-y)
$$

The vibrational distribution is usually much narrower than the electronic distribution. When the transition frequencies of the two vibronic transitions are fully correlated, the vibrational distribution function approaches the Dirac $\delta$ function. The hole spectrum is defined as the difference of the absorption spectra:

$$
H\left(\omega, \omega_{B}\right)=A_{0}(\omega)-A\left(\omega, \omega_{B}\right)
$$

When eq 11 is valid, the spectra can be written in the form

$$
H\left(\omega, \omega_{B}\right)=\int \mathrm{d} z g_{v}(z) H^{c}\left(\omega-z, \omega_{B}\right)
$$




$$
\begin{gathered}
H^{c}\left(\omega, \omega_{B}\right)=\int \mathrm{d} \Omega / 8 \pi^{2} \int \mathrm{d} x g_{e}(x) \Delta N_{a}\left(\omega_{B}-\right. \\
x, \cos \beta) \sigma_{a c}(\omega-x)\left|\mathbf{e}_{P} \mathbf{e}_{a c}\right|^{2} \\
A_{0}^{c}(\omega)=1 / 3 \int \mathrm{d} x g_{e}(x) \sigma_{a c}(\omega-x) \\
\Delta N_{a}=1-N_{a}
\end{gathered}
$$

Here $H^{c}\left(\omega, \omega_{B}\right)$ and $A_{0}^{c}(\omega)$ apply to the case of full correlation. In the following we restrict our considerations to this fully correlated case since according to eq 13 the less correlated case requires only a convolution of the final result with the vibrational distribution function.

The orientational average can be considerably simplified (see, e.g., ref 7) with the help of the relation

$$
\begin{aligned}
\int \mathrm{d} \Omega / 8 \pi^{2} f\left(\left|\mathbf{e}_{B} \mathbf{e}_{a b}\right|^{2}\right)\left|\mathbf{e}_{P} \mathbf{e}_{a d}\right|^{2} & =F_{0}+ \\
& \left(3 F_{2}-F_{0}\right) P_{2}\left(\mathbf{e}_{B} \mathbf{e}_{P}\right) P_{2}\left(\mathbf{e}_{a b} \mathbf{e}_{a c}\right)
\end{aligned}
$$

with the Legendre polynomials $P_{2}(x)=\left(3 x^{2}-1\right) / 2$ and

$$
F_{n}=1 / 3 \int_{0}^{1} \mathrm{~d} q q^{n} f\left(q^{2}\right)
$$

Hence only a one-dimensional integration over $q=\cos \beta$ remains, and the result is valid for all possible mutual orientations of the light polarizations and the transition dipole moments. For the case of the hole spectrum of the fully correlated ensemble the functions $F_{n}$ are given by

$$
F_{n}\left(\omega, \omega_{B}\right)=1 / 3 \int \mathrm{d} x \int_{0}^{1} \mathrm{~d} q q^{n} g_{e}(x) \Delta N_{a}\left(\omega_{B}-x, q\right) \sigma_{a c}(\omega-x)
$$

The function $F_{0}$ is the isotropic contribution to the line shape of the hole, which is measured when $e_{B}$ and $e_{P}$ form the magic angle of $54.7^{\circ}$. For a resonant hole $\left(e_{a b}=e_{a c}\right)$ the line shape measured with parallel polarization of burning and probing light is $H_{\|}^{c}=$ $3 F_{2}$ and with perpendicular polarization $H_{1}^{c}=3\left(F_{0}-F_{2}\right) / 2$.

2.2. Limit of a Broad Inhomogeneous Distribution. Before eq 19 can be evaluated, reasonable model assumptions have to be made regarding the inhomogeneous distribution function, the homogeneous line shape, and the photochemical change in the population of the ground state. The inhomogeneous distribution is in many cases well approximated by a Gaussian, but a knowledge of its particular form is not even necessary when the homogeneous line shape is much narrower. Then the integrand in eq 19 is different from zero only in a small region around $x \approx x_{B}=\omega_{B}$ $-\omega_{b a}^{0}$. The inhomogeneous line-shape function can be considered constant over this range and taken outside the integral:

$$
\begin{array}{r}
F_{n}\left(\omega, \omega_{B}\right)=1 / 3 g_{e}\left(\omega_{B}-\omega_{b a}^{0}\right) \int \mathrm{d} x \int_{0}^{1} \mathrm{~d} q q^{n} \Delta N_{a}\left(\omega_{B}-\right. \\
x, q) \sigma_{a c}(\omega-x)
\end{array}
$$

The homogeneous line shape consists of a Lorentzian zero-phonon line $L$ and a phonon sideband $P$ :

$$
\begin{gathered}
\sigma_{a c}(\omega)=\sigma_{a c}^{0}\left[f_{a c} L\left(\omega-\omega_{c a}^{0}, \Gamma_{a c}\right)+\right. \\
\left.\left(1-f_{a c}\right) P\left(\omega-\omega_{c a}^{0}-\Delta_{c a}, S_{a c}\right)\right] \\
L(\omega, \Gamma)=\frac{\Gamma / \pi}{\omega^{2}+\Gamma^{2}}
\end{gathered}
$$

Here $f_{a c}$ is the Debye-Waller factor, and both line-shape functions $L$ and $P$ are normalized to unit integral. Hence, $\sigma_{a c}^{0}$ is the integrated absorption cross section, and $\sigma_{a a}^{0} f_{a c}$ the area under the zero-phonon line. The phonon sideband has width $S_{a c}$ and its maximum is shifted by $\Delta_{c a}$ with respect to the zero-phonon line. It is often approximated by a Gaussian. We will not assume any particular form but use only the fact that its width is much larger than $\Gamma_{a c}$. The hole-shape functions due to the zero-phonon lines, which we want to label $F_{n}^{2}$, are then readily distinguished from the broad contributions arising from the phonon sidebands.

If $g_{e}(x)$ is much broader than $\sigma_{a c}(\omega)$ (including the phonon sideband), the technique to derive eq 20 can also be applied to the absorption spectrum before hole burning, with the result

$$
A_{0}(\omega)=1 / 3 g_{e}\left(\omega-\omega_{b a}^{0}\right) \sigma_{a c}^{0}
$$

This opens a way for the determination of the integrated homogeneous cross section by integrating over the inhomogeneous absorption spectrum:

$$
\sigma_{a c}^{0}=3 \int \mathrm{d} \omega A_{0}(\omega)
$$

The factor 3 arises since $\sigma_{\alpha c}^{0}$ refers to a molecule with transition dipole parallel to the light polarization, whereas $A_{0}(\omega)$ is the average cross section for the isotropic ensemble. Equation 23 can be used to normalize the hole-shape functions:

$$
\begin{aligned}
\hat{F}_{n}^{2}\left(\omega, \omega_{B}\right) & =F_{n}^{2}\left(\omega, \omega_{B}\right) / A_{0}\left(\omega_{B}\right) \\
& =f_{a c} \int \mathrm{d} x \int_{0}^{1} \mathrm{~d} q q^{n} \Delta N_{a}\left(\omega_{B}-x, q\right) \times \\
& \frac{\Gamma_{a c} / \pi}{\left(\omega-x-\omega_{c a}^{0}\right)^{2}+\Gamma_{a c}{ }^{2}}
\end{aligned}
$$

Hence it is seen that in the infinite burning limit, when $\Delta N_{a}=$ 1 , eq 25 reduces to $\hat{F}_{n}^{2}=f_{a c} /(n+1)$ leading to $\hat{H}=f_{a c}$ for all polarizations. Therefore, under the conditions given above the asymptotic value of the relative hole depth can be interpreted as the Debye-Waller factor. In practical cases the condition necessary for the validity of eq 23 may not be fulfilled, since the shift $\Delta_{c a}$ of the phonon sideband is often not small compared to the inhomogeneous width. The contribution from phonon sidebands to the inhomogeneous absorption band is smaller at the red edge and larger at the blue edge. This introduces an error into the determination of the Debye-Waller factor through extrapolation of the hole depth especially if $f_{a c}$ is small. The asymptotic value of the hole depth should then be treated as an adjustable parameter indicating the "burnable" part of the absorption.

Evaluation of the hole depth often requires deconvolution of the observed hole shapes with a spectrometer function. If the width of the hole and the spectral resolution are of comparable size it might be more convenient to evaluate the area under the normalized hole:

$$
I_{n}=\int \mathrm{d} \omega \hat{F}_{n}^{z}\left(\omega, \omega_{B}\right)=f_{a c} \int \mathrm{d} x \int_{0}^{1} \mathrm{~d} q q^{n} \Delta N_{a}\left(\omega_{B}-x, q\right)
$$

This requires no knowledge of the homogeneous line-width parameter.

2.3. Low-Intensity (cw) Hole-Burning. Hole burning with $\mathrm{cw}$ light can be modeled as a unimolecular decay with rate constant $k_{X} \Phi$, where $k_{X}$ is the excitation rate given by eq 4 and $\Phi$ is the quantum yield of the photochemical reaction. In the level system shown in Figure 1 the photoproduct state $r$ can be populated from the optically pumped level $b$ either directly or via the intermediate level $d$. This leads to the following expression for the photochemical quantum yield:

$$
\Phi=\frac{\gamma_{b d}}{\gamma_{b a}+\gamma_{b d}+\gamma_{b r}} \frac{\gamma_{d r}}{\gamma_{d r}+\gamma_{d a}}+\frac{\gamma_{b r}}{\gamma_{b a}+\gamma_{b d}+\gamma_{b r}}
$$

The first term accounts for the indirect decay channel. For most 
hole-burning reactions the photochemical quantum yield is much smaller than 1 , with the necessary consequence $\gamma_{b r} \ll \gamma_{b a}+\gamma_{b d}$. In addition at least one of the inequalities $\gamma_{b a} \gg \gamma_{b d}$ and $\gamma_{d a} \gg$ $\gamma_{d r}$ must be true. The expression for $\Delta N$ in the cw model is

$$
\Delta N_{a}^{c w}\left(\omega_{B}-x, q\right)=1-\exp \left[-\Phi \sigma_{a b}\left(\omega_{B}-x\right) F_{B} t q^{2}\right]
$$

for the change in population. The absorption cross section $\sigma_{a b}(\omega)$ may be described by an ansatz analogous to eq 21 , and for the modeling of the zero-phonon hole the phonon sideband is usually neglected. In the limit of small burning fluence $F_{B} t$ the exponential can be expanded, resulting in decoupling of the integrals over frequency and orientation:

$$
\begin{gathered}
\Delta N_{a}^{c w}\left(\omega_{B}-x\right) \approx \Phi f_{a b} \sigma_{a b}^{0} L\left(\omega_{B}-x, \Gamma_{a b}\right) F_{B} t q^{2} \\
\hat{F}_{n}^{a}\left(\omega, \omega_{B}\right)=\frac{1}{n+3} L\left(\omega-\omega_{B}, \Gamma_{a b}+\Gamma_{a c}\right) f_{a b} f_{a c} \Phi \sigma_{a b}^{0} F_{B} t
\end{gathered}
$$

Hence in this limit for all mutual orientations of polarizers and transition moments the same line shape is observed, namely, a Lorentzian centered at $\omega=\omega_{B}$ with the sum of the two homogeneous line widths. Insertion of eq 30 into eq 17 leads to Perrin's formula ${ }^{1}$ for the degree of polarization $\rho$ of the spectral hole. For larger burning fluence eq 28 can no longer be expanded and has to be integrated. The integration over the orientational variable $q$ can be performed with the help of

$$
\begin{gathered}
\int_{0}^{1} \mathrm{~d} q \exp \left(-a q^{2}\right)=1 / 2 \sqrt{\pi / a} \operatorname{erf}(\sqrt{a}) \\
\int_{0}^{1} \mathrm{~d} q q^{2} \exp \left(-a q^{2}\right)=(1 / 4 a)[\sqrt{\pi / a} \operatorname{erf}(\sqrt{a})-2 \exp (-a)]
\end{gathered}
$$

The remaining integral over frequency was performed numerically.

It should be noted that within the cw model the formula for the hole depth contains only two effective parameters, namely, $f_{a c}$ and $Y=\Phi f_{a b} \sigma_{a b}^{0} F_{B} / \Gamma_{a b}$. The plot of $\rho$ versus hole depth will only depend on $f_{a c}$ and the angle $\delta$ between the transition dipoles, but not on $\Phi$ or $\sigma_{a b}^{0}$. A fit to the saturation of the hole depth versus burning time will yield the parameter $Y$ and the DebyeWaller factor $f_{a c}$. The parameter $Y$ can also be obtained from the initial growth rate of the depth of the resonant hole:

$$
\frac{1}{A_{0}\left(\omega_{B}\right)} \frac{\mathrm{d} H_{\|}}{\mathrm{d} t}=\frac{3}{10 \pi} f_{a b} Y
$$

The parameter $\sigma_{a b}^{0}$ can be obtained from the integrated absorption spectrum (eq 24), and $\Gamma_{a b}$ is one-quarter of the hole width at early burning times. The photochemical quantum yield $\Phi$ can then be calculated if the photon flux $F_{B}$ can be measured with sufficient accuracy.

2.4. Hole Burning with Intense Pulsed Lasers. In hole-burning experiments with pulsed laser light it was observed that the degree of polarization $\rho$ of the resonant hole did not extrapolate to $1 / 2$ for zero fluence. This can be rationalized by the assumption that a considerable fraction of the resonant molecular ensemble is pumped to the excited state by a single laser pulse. If the photochemical quantum yield is high, this will immediately lead to a deep hole with corresponding loss of anisotropy. Due to a small quantum yield, however, only a small contribution to the hole is produced, but the anisotropy stored is that of the initially excited subensemble.

This was modeled by integration of the rate equations corresponding to the level system shown in Figure 1 with a short light pulse of duration $\tau$. This generates the populations $N_{b}(\tau)$, $N_{d}(\tau)$, and $N_{r}(\tau)$ which successively decay to the levels $a$ or $r$. The amount finally removed from the ground state by a single laser pulse is

$$
\delta N_{a}=\Phi N_{b}(\tau)+\tilde{\Phi} N_{d}(\tau)+N_{r}(\tau)
$$

with $\tilde{\Phi}=\gamma_{d r} /\left(\gamma_{d r}+\gamma_{d a}\right)$. The resulting fractional change $\delta N_{a} /$ $N_{a}$ in the ground-state population may be interpreted as the product of $\Phi$ with an effective excitation yield per laser pulse $k_{X}^{e}$. The analytical solution of the complete rate equation system is rather involved and will not be reproduced here. It was performed with the help of the computer algebra system REDUCE.8 REDUCE also transforms the result into a FORTRAN subroutine which was incorporated into our numerical code for simulations and fits.

If the lifetime of the intermediate level $d$ is long compared to the pulse duration $\tau$, the populations $N_{d}(\tau)$ and $N_{r}(\tau)$ are generated solely through decay from level $b$, i.e.

$$
\begin{aligned}
& N_{r}(\tau)=\gamma_{b r} \int_{0}^{\tau} \mathrm{d} t N_{b}(t) \\
& N_{d}(\tau)=\gamma_{b d} \int_{0}^{\tau} \mathrm{d} t N_{b}(t)
\end{aligned}
$$

Hence eq 34 has the form

$$
\begin{gathered}
\delta N_{a}=\Phi N_{b}(\tau)+\hat{\Phi} \int_{0}^{\tau} \mathrm{d} t N_{b}(t) \\
\hat{\Phi}=\tilde{\Phi} \gamma_{b d}+\gamma_{b r}
\end{gathered}
$$

Comparison with eq 27 shows that $\Phi=\Phi / \tau_{b}$, where $\tau_{b}=1 /\left(\gamma_{b a}\right.$ $\left.+\gamma_{b d}+\gamma_{b r}\right)$ is the lifetime of level $b$. This means that for a long-lived intermediate state the result is independent from the branching ratio $\gamma_{b r} / \gamma_{b d}$ and hence the direct path of photochemical hole burning and the indirect path via the level $d$ cannot be distinguished.

The analytical expressions simplify considerably if the population of the intermediate level $d$ and the product level $r$ can be neclected during the pulse duration $\tau$. This is likely if the pulse duration is short so that $\left(\gamma_{b d}+\gamma_{b r}\right) \tau \ll 1$ (case 1$)$. Only the rate equations of the two-level system $a b$ must be solved with the result

$$
\frac{\delta N_{a}}{N_{a}}=\Phi k_{X}^{e}=\frac{\Phi k_{X}}{2 k_{X}+\gamma_{b a}}\left[1-\exp \left\{-\left(2 k_{X}+\gamma_{b a}\right) \tau\right\}\right]
$$

If the decay to the intermediate level $d$ is the fastest rate constant $\left(\gamma_{b d} \gg k_{X}\right)$ so that $N_{b} \approx 0$ during the pump pulse and during this time ground-state recovery through $\gamma_{d a}$ can be neglected (case 2 ), the effective hole-burning yield per laser pulse is

$$
\frac{\delta N_{a}}{N_{a}}=\Phi k_{X}^{e}=\tilde{\Phi}\left[1-\exp \left\{-\frac{k_{X} \gamma_{b d} \tau}{\gamma_{b d}+\gamma_{b a}}\right\}\right]
$$

In either case the change in the population after $n_{P}$ laser pulses is given by

$$
\Delta N_{a}=1-\exp \left[-n_{P} \Phi k_{X}^{e}\right]
$$

If $\gamma_{b a} \ll 2 k_{X}$ applies in case 1 , both equations have the form

$$
\Delta N_{a}=1-\exp \left[-\frac{n_{P}}{b} \Phi\left(1-\exp \left[-b k_{X} \tau\right]\right)\right]
$$

where $b=2$ for case 1 and $b=\gamma_{b d} /\left(\gamma_{b a}+\gamma_{b d}+\gamma_{b r}\right)$ for case 2 , the latter quantity being the triplet quantum yield $\Phi_{T}$ if $d$ is the triplet level. It is seen that for small pump rate $k_{X}$ eq 42 reduces to the $\mathrm{cw}$ case (eq 28) with $n_{P}=t$ being the total irradiation time. If $k_{x}$ is of the same order of magnitude or even larger than $\gamma_{b d}$, a simplified form like eq 42 cannot be found. Therefore, we have mostly used the FORTRAN subroutine for 


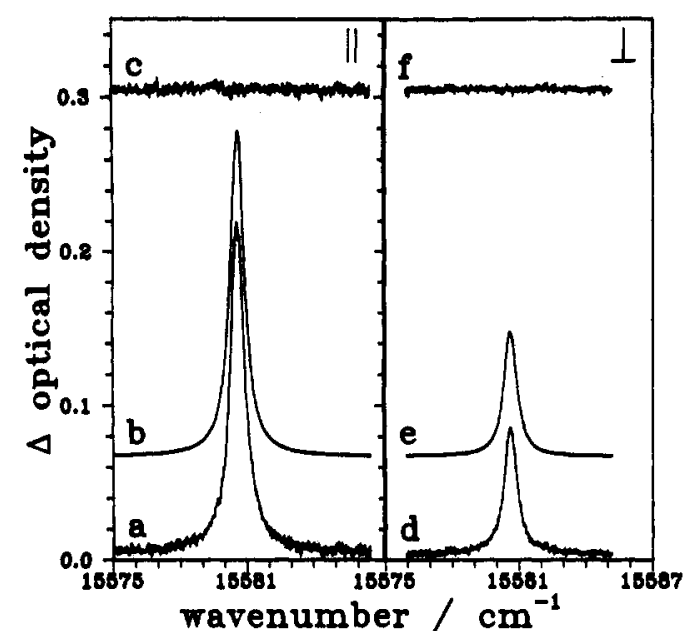

Flgure 2. Profiles of a resonant hole burnt into the electronic origin band of TPP in PMMA at $T=10 \mathrm{~K}$ : (a) observed with parallel polarization of burning and probe light; (b) fit of a Voigt profile with $B_{G}=0.21 \mathrm{~cm}^{-1}$ and $B_{L}=0.61 \mathrm{~cm}^{-1} ;$ (c) residuals; $(d-f)$ corresponding data for perpendicularly polarized burning and probing light.

the complete rate equation system and solved the two dimensional integration of eq 25 numerically.

\section{Experimental Section}

A solution of meso-tetraphenylporphine (TPP) in methyl methacrylate was thermally polymerized with azabisisobutyronitrile as radical starter (see ref 9 for details). Slices of 1.5$\mathrm{mm}$ thickness were optically polished and glued onto a $\mathrm{LiF}$ window mounted to the coldfinger of a closed-cycle refrigerator cryostate (Leybold ROK 10-300). This cryostate was modified by incorporating quartz windows into the heat shield which effectively block thermal radiation from the sample. Spectral holes were burnt with an excimer laser pumped pulsed dye laser (Lambda Physik EMG 101 and LPD 3000) with a line width of ca. $0.1 \mathrm{~cm}^{-1}$ as checked with a monitor etalon. Only the central part of the beam was selected with an aperture. The path of the hole-burning laser was made collinear with that of the probe light by means of a moveable mirror. The same Glan polarizer could thus be used to polarize either the burning or the probing light. Absorption spectra were measured with the light of a high-pressure xenon arclamp (XBO 75) dispersed by a double-grating monochromator (Spex 1404) with a resolution of $0.08 \AA\left(0.2 \mathrm{~cm}^{-1}\right)$. The beam leaving the monochromator was split into a signal and a reference beam by a glass plate. The intensities of the transmitted signal beam and the reference beam were measured by photon counting. The resulting signal-to-noise ratio corresponds to an accuracy of 0.002 optical density units.

\section{Results and Discussion}

4.1. Resonant Holes at Low Burning Intensity. Narrowbandwidth excitation at $10 \mathrm{~K}$ into the lowest energy absorption band of TPP in PMMA (center wavenumber $15576 \mathrm{~cm}^{-1}$ ) leads to permanent spectral holes. The photochemical process can be attributed to a tautomerization of the two inner protons as in the parent compound porphine. Figure 2 shows the profiles of a resonant hole observed with the probe light polarized parallel (curve a) and perpendicular (curve d) to the polarization of the hole-burning light. Fits of a Voigt profile with the Gaussian line-width parameter fixed at the spectrometer resolution $(0.21$ $\mathrm{cm}^{-1}$ ) are shown as curves $b$ and $e$. As judged from the residues (curves $c$ and $f$ ) the agreement with experiment is excellent. The Lorentzian line-width parameter of the fit is $B_{L}=0.61 \mathrm{~cm}^{-1}$. From the relative hole depths $H_{1}=0.149$ and $H_{\perp}=0.058$ the polarization degree $\rho=0.44$ is obtained, a value significantly lower than the value $\rho=0.5$ expected for a shallow resonant hole.

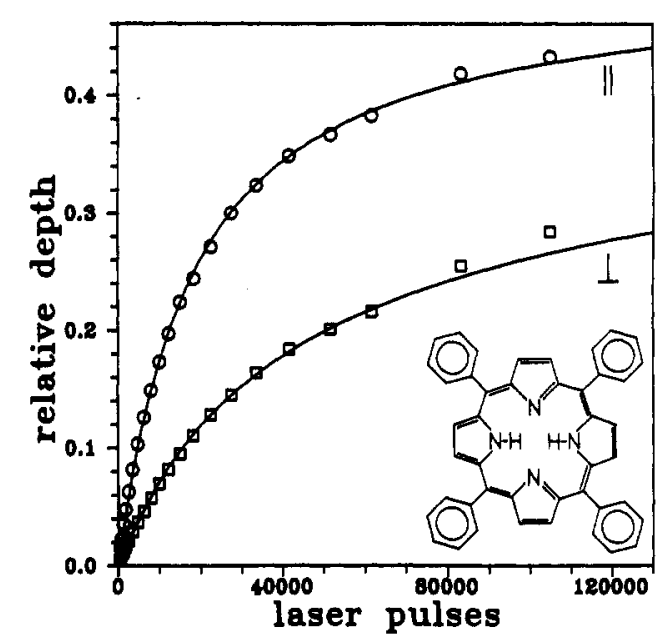

Figure 3. Saturation of the relative hole depth of the resonant spectral hole in the electronic origin band of TPP in PMMA at $T=10 \mathrm{~K}$. Circles and squares refer to $H_{\|}$and $H_{\perp}$. One laser pulse corresponds to a fluence of $I \tau=0.67 \mu \mathrm{J} \mathrm{cm}^{-2}$. Upper curve: fit of eq 28 (four-layer model) to the data for $H_{\|}$with $f_{a b}=0.54$ and $Y_{\tau}=\Phi f_{a b} \sigma_{a b}^{0} F_{B} \tau / \Gamma_{a b}=1.2 \times 10^{-3}$. Lower curve: simulation with the same parameters for $H_{\perp}$.

Figure 3 shows the observed saturation behavior for the relative hole depths $H_{\sharp}$ and $H_{\perp}$ as a function of the number of laser pulses used for burning. The fluence delivered with each laser pulse of 8-ns duration was ca. $0.67 \mu \mathrm{J} / \mathrm{cm}^{2}$, corresponding to an intensity of $84 \mathrm{~W} / \mathrm{cm}^{2}$. A fit of the $\mathrm{cw}$ model (eqs 25 and 28 ) to $H_{\|}$is shown as the upper solid line in Figure 3. Since the initial optical density at the burning frequency was 1.5 , attenuation of the burning beam was not negligible. In the numerical calculation the sample was approximated by a sequence of four optically thin layers. The burning intensity for each layer was taken as the fraction transmitted by the preceding layers. The parameters obtained from the fit are the Debye-Waller factor $f_{a b}=0.54 \pm$ 0.06 and $Y \tau=\Phi f_{a b} \sigma_{a b}^{0} F_{B} \tau / \Gamma_{a b}=1.2 \times 10^{-3}$. The lower solid line in Figure 3 is a simulation for $H_{\perp}$ with these same parameters. It is obvious that the same parameters describe both saturation curves very well. Hence the saturation of $\rho$ is also well described (see Figure 4 below). Similar experiments performed at other temperatures revealed a small temperature dependence of the Debye-Waller factor. The values obtained were $f_{a b}=0.42 \pm$ 0.05 at $T=16 \mathrm{~K}$ and $f_{a b}=0.40 \pm 0.05$ at $T=21 \mathrm{~K}$.

Integration of the first absorption band of TPP according to eq 24 yields $\sigma^{0}=4.4 \times 10^{-3} \mathrm{~cm}^{2} / \mathrm{s}$. From the fluence given above a dose of $F_{B} \tau=2.2 \times 10^{12}$ photons $/ \mathrm{cm}^{2}$ and pulse is calculated. Together with $\Gamma_{a b}=0.12 \mathrm{~cm}^{-1}$ obtained from the width of the holes at lowest fluences this leads to $\Phi=6 \times 10^{-3}$. The main source of error in this value is the uncertainty in the absolute value of the photon flux.

4.2. Effect of High Burning Intensity. When hole burning was performed with pulses of higher intensity, considerable deviations from the $\mathrm{cw}$ model were observed. The relative hole depth still extrapolated to the Debye-Waller factor, but the polarization degree indicated a considerable bleaching of the anisotropy of the spectral holes even in the limit of zero fluence. This is exemplified in Figure 4, where the result of a low-intensity experiment (circles, $I \tau \approx 0.67 \mu \mathrm{J} / \mathrm{cm}^{2}$ ) is compared to two experiments with higher intensity (squares, $I \tau \approx 13 \mu \mathrm{J} / \mathrm{cm}^{2}$; triangles, $I_{\tau} \approx 24 \mu \mathrm{J} / \mathrm{cm}^{2}$ ). The dashed line in Figure 4 is a simulation based on the cw model for an optically thin sample; curve a was calculated for a sample with optical density 1.5 approximated by four layers. Only the first set of experimental data agrees with the prediction of the $\mathrm{cw}$ model. Note that within the cw model the plot $\rho$ vs $H_{\|}$does not depend on any of the parameters $\Phi, \sigma_{a b}^{0}, \Gamma_{a b}, F_{B}$, or $\tau$.

The loss of anisotropy could be the result of orientational or spectral diffusion caused by heating of the sample. An upper 


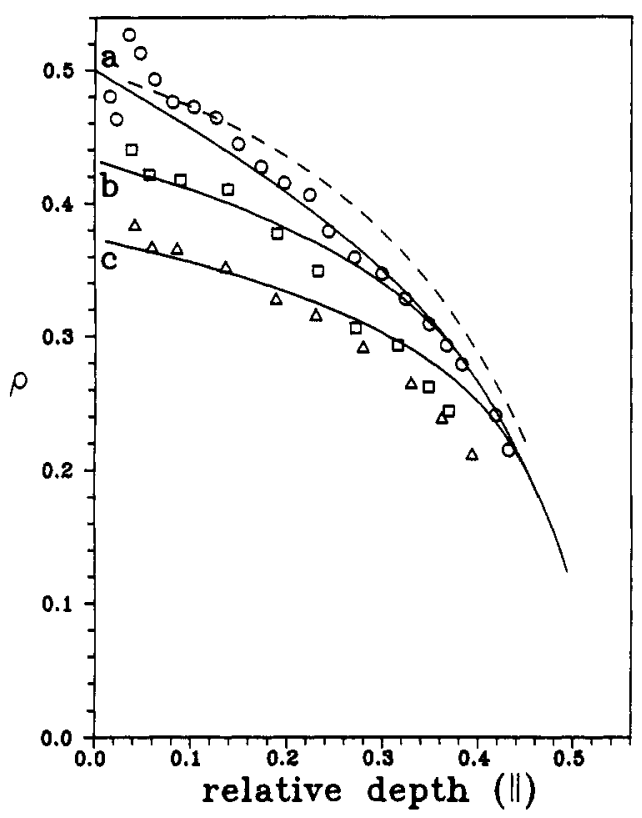

Figure 4. Saturation of the degree of polarization $\rho$ with increasing relative hole depth. Experimental data points refer to the resonant spectral hole in the electronic origin band of TPP in PMMA burnt with fluences per laser pulse of $0.67 \mu \mathrm{J} \mathrm{cm}^{-2}$ (circles), $13 \mu \mathrm{J} \mathrm{cm}^{-2}$ (squares), and $24 \mu \mathrm{J} \mathrm{cm}-2$ (triangles). Simulations: cw model of hole burning for an optically thin sample (dashed line) and an optically thick sample approximated by four layers (curve a). Curves $b$ and $c$ are fits of the high pulse-power model of spectral hole burning (eq 41) to the data from higher fluences.

limit for the temperature increase produced by the absorbed burning light energy $Q$ is $\Delta T=Q / C_{p}$, where $C_{p}=2.1 \times 10^{-2} \mathrm{~W}$ $s \mathrm{~g}^{-1} \mathrm{~K}^{-1}$ is the heat capacity of PMMA at a temperature of 10 K. ${ }^{10}$ This neglects any heat diffusion out of the irradiated volume during one hole-burning sequence which may consist of several hundred laser pulses. We estimate $\Delta T<0.5 \mathrm{~K}$ for the first six points in Figure 4, and $\Delta T<3 \mathrm{~K}$ for the last points. Since at a temperature of $13 \mathrm{~K}$ the low-intensity hole-burning response of the system is well described by the cw model, a contribution of orientational or spectral diffusion to the loss of anisotropy is unlikely.

An alternative explanation is given by the model of hole burning with intense laser pulses presented in section 2.4. In the case of TPP the states $a$ and $r$ correspond to the two ground-state tautomers. For the resonant spectral holes considered here both state labels $b$ and $c$ refer to the vibrationless level of the first excited singlet state $S_{1}$, and $d$ refers to the first triplet level $T_{1}$. The lifetime of $S_{1}$ is known $\left(\tau\left(S_{1}\right)=11.6 \mathrm{~ns}^{11}\right)$ leading to $\gamma_{b a}+$ $\gamma_{b d}+\gamma_{b r}=8.62 \times 10^{7} \mathrm{~s}^{-1}$. With the quantum yield of triplet formation $\left(\Phi_{T}=0.80^{12,13}\right)$ the rate constant $\gamma_{b d}=6.90 \times 10^{7} \mathrm{~s}^{-1}$ for ISC can be isolated. Since the quantum yield of photochemical tautomerization is much smaller than unity it follows that $\gamma_{b r} \ll$ $\gamma_{b a} \approx 1.7 \times 10^{7} \mathrm{~s}^{-1}$. Finally, from the triplet lifetime $\tau\left(\mathrm{T}_{1}\right)=$ $1.4 \mathrm{~ms}$, it follows that $\gamma_{d a} \leq 700 \mathrm{~s}^{-1}$, and hence this decay channel may be neglected on the time scale of a laser pulse. With the Debye-Waller factor fixed at 0.54 the only remaining parameters of the model are the photochemical quantum yield $\Phi$ and the excitation rate $k_{X}^{0}=\sigma\left(\omega_{b a}^{0}\right) F_{B}$ for molecules with their transition dipole parallel to the light polarization.

A simultaneous fit of the model to the experimental data for $\rho$ presented in Figure 4 and the corresponding data for $H_{\|}$yielded the parameters collected in the last two columns of Table 1 . The excitation rate $k_{X}^{0}$ can also be calculated from the applied laser intensity, and these values (column 2 of Table 1) are in good agreement with those from the fit. Furthermore, the value of the quantum yield agrees well with that obtained within the cw model.

Laser pulses that bleach out a considerable portion of a homogeneous subensemble must also lead to power broadening
TABLE 1: Quantum Yield of Photochemical Reaction $\Phi$ and Excitation Rate $k_{X}^{0}$ Obtained from a Fit of the $\mathrm{Cw}$ Model or the Pulse Model to the Data $H_{1}$ and $\rho$ from Three Experiments with Increasing Fluence $I_{T}=F_{B} h \omega_{B} \tau$ per Laser Pulse'

\begin{tabular}{cccccc}
\hline \multicolumn{2}{c}{ experiment } & \multicolumn{3}{c}{ fit } \\
\cline { 5 - 6 }$I \tau / \mu \mathrm{J} \mathrm{cm}^{-2}$ & $k_{x}^{0} / 10^{9} \mathrm{~s}^{-1}$ & & $10^{3} \Phi$ & $k_{x}^{0} / 10^{9} \mathrm{~s}^{-1}$ & model \\
\hline 0.67 & 0.009 & & 6.0 & & $\mathrm{cw}$ \\
13 & 0.17 & & 6.3 & 0.18 & pulse \\
24 & 0.32 & & 7.2 & 0.43 & pulse
\end{tabular}

a The second column gives values for $k_{X}^{0}$ calculated from the fluence and the peak absorption cross section of the zero phonon line.

TABLE 2: Power Broadening of the Hole Widths $B_{\|}$and $B_{\perp}$ (Accurate to $\pm 0.02 \mathrm{~cm}^{-1}$ ) Extrapolated to Zero Hole Depthe

\begin{tabular}{ccccccc}
\hline $\begin{array}{c}I \tau / \mu \mathrm{J} \\
\mathrm{cm}^{-2}\end{array}$ & $\begin{array}{c}f \omega_{R}{ }^{2} T_{1} T_{2} \\
(\beta=0)\end{array}$ & $\begin{array}{c}B_{\|} / \\
\mathrm{cm}^{-1}\end{array}$ & $\begin{array}{c}B_{\perp} / \\
\mathrm{cm}^{-1}\end{array}$ & $\begin{array}{c}2 \Gamma_{P} / \\
\mathrm{cm}^{-1}\end{array}$ & $\Gamma_{P} / \Gamma$ & $\left(\omega_{\omega_{R}}{ }^{2} T_{1} T_{2}\right)$ \\
\hline 0.67 & 0.052 & 0.49 & 0.48 & & & \\
13 & 1.9 & 0.56 & 0.48 & 0.32 & 1.33 & 0.77 \\
24 & 3.5 & 0.58 & 0.53 & 0.34 & 1.42 & 1.0
\end{tabular}

- The first two columns give the fluence per pulse for each experiment and the estimated maximum broadening parameter for aligned molecules. The last column gives the orientationally averaged broadening parameter calculated from $\Gamma_{P} / \Gamma$.

TABLE 3: Line-Width Parameters of Three Satellite Holes in $\mathbf{c m}^{-1}$ •

\begin{tabular}{ccccc}
\hline & $\begin{array}{c}\text { width of } \\
\bar{\nu}_{\text {vib }}\end{array}$ & $\begin{array}{c}2 \Gamma_{a b}= \\
\text { Voigt curve }\end{array}$ & $\begin{array}{c}2 \Gamma_{L b} \\
\text { resonant }\end{array}$ & \\
\hline 955 & $B_{G}=3.1$ & 1.1 & 1.4 & 3.1 \\
& $B_{L}=1.3$ & & & \\
1194 & $B_{V}=8.0$ & & 3.0 & 6.0 \\
1207 & $B_{V}=9.7$ & & 3.0 & 7.8
\end{tabular}

a $B_{G}, B_{L}$, and $B_{V}$ are the Gaussian, the Lorentzian, and the total width of the Voigt function fitted to the satellite hole. $2 \Gamma_{a b}$ is the homogeneous line width of the vibronic transition. $B_{\text {vib }}$ is the Gaussian width of the inhomogeneous distribution of the vibrational frequencies. The error margins are $\pm 0.2 \mathrm{~cm}^{-1}$.

of the homogeneous line width which should be observable in the widths of the spectral holes. Under steady-state excitation of a three-level system power broadening leads to an effective homogeneous line width parameter of the excited transition given by 5

$$
\Gamma_{P}=\Gamma\left[1+f_{a b} \omega_{R}^{2} T_{1} T_{2}\left(2+\gamma_{b d} / \gamma_{d a}\right) / 2\right]^{1 / 2}
$$

where $T_{1}$ is the lifetime of the upper state, $T_{2}=1 / \Gamma$ the dephasing time of the level pair, and $\omega_{R}$ the Rabi frequency defined by

$$
\hbar \omega_{R}=\mu E \cos \beta
$$

The factor $\left(2+\gamma_{b d} / \gamma_{d a}\right) / 2$ should be of minor importance in our case since the steady state is never achieved during one laser pulse. The width of a spectral hole burnt into an aligned ensemble of molecules is then $B=2\left(\Gamma_{P}+\Gamma\right)$. In an isotropic ensemble each orientational subensemble will experience a different Rabi frequency, and the hole width will represent an average of the broadening parameter $f_{a b} \omega_{R}^{2} T_{1} T_{2}$. To extract these from the experiment, the hole width was first extrapolated to zero hole depth. This removes the contribution from photochemical saturation to the width. The parameters obtained for three experiments with increasing laser intensity are collected in Table 2. For the highest laser intensity an averaged broadening parameter of 1.0 is obtained (last column), corresponding to an increase of the homogeneous line width by $42 \%$ and of the spectral hole by $21 \%$. Power broadening affects the width of $H_{\|}$more than that of $H_{\perp}$, which is in line with the fact that the Rabi frequency is largest for the set of molecules with their transition 
dipole parallel to the light polarization. The broadening parameter of this subensemble can be estimated from the applied laser intensity and the transition dipole $\mu$. A value of $\mu=0.94 \mathrm{D}$ (3.1 $\times 10^{-30} \mathrm{C} \mathrm{m}$ ) was obtained from the integral over the region of the electronic origin of the first transition in TPP. The maximum broadening parameters calculated in this way are collected in column 2 of Table 2 . Since the Rabi frequency is proportional to $\cos \beta$, a first order estimate of the average broadening parameter yields one-third of the maximum value, in good agreement with the value obtained from the line widths.

4.3. Debye-Waller Factor and Photochemical Quantum Yield. In this work a Debye-Waller factor $f=0.54 \pm 0.06$ and a homogeneous line width $2 \Gamma=0.24 \mathrm{~cm}^{-1}$ were found for the longest wavelength absorption band of TPP in PMMA at $10 \mathrm{~K}$. In an accumulated photon echo experiment on the same system at the same temperature the values $f=0.8$ and $2 \Gamma=0.5 \mathrm{~cm}^{-1}$ were obtained. ${ }^{14}$ Hole burning and accumulated photon echo experiments both suffer from spectral diffusion. Spectral diffusion leads to smaller hole depth and hence an apparently smaller Debye-Waller factor but also to an increased hole width. Since we observe a narrower hole and a smaller Debye-Waller factor, spectral diffusion can be ruled out as the source of the discrepancy. From hole-burning experiments on a similar system, phthalocyanine in PMMA, the Debye-Waller factors $f=0.77 \pm 0.1$ at $T \leq 12 \mathrm{~K}$ and $f=0.57 \pm 0.1$ at $T=20 \mathrm{~K}$ were reported. ${ }^{4} \mathrm{In}$ our investigation on tetraphenylporphine the same reduction of the Debye-Waller factor by ca. $25 \%$ was observed in going from 10 to $20 \mathrm{~K}$. In both systems the hole-burning process involves tautomerization of the inner protons, and the same temperature dependence of the Debye-Waller factor could indicate similar electron-phonon coupling in both compounds. However, there is no a priori reason why the Debye-Waller factors should be equal. Nevertheless, our Debye-Waller factors seem to be systematically lower than literature values for the same or similar compounds. This could be caused by a small temperature gradient between the sample and the temperature sensor. Our sample is cooled not by direct contact with $\mathrm{He}$ gas but only through mechanical contact with a coldfinger. Hence the temperature of the sample might be slightly higher than that of the heat exchanger.

In some papers a quantum yield of hole formation $\Phi_{B}$ is defined ${ }^{15-18}$ as the number of chromophores contributing to the hole divided by the number of photons absorbed by each chromophore. The relative change of the hole area is taken as measure of the number of chromophores. Hence

$$
\Phi_{B}=\left(\frac{\mathrm{d}}{\mathrm{d} t} \int H_{\|}(\omega) \mathrm{d} \omega\right)_{t=0}\left(\int A_{0}(\omega) \mathrm{d} \omega\right)^{-1} \frac{N l}{F_{B}\left(1-T_{0}\right)}
$$

where $T_{0}=\exp \left(-A_{0}\left(\omega_{B}\right) N l\right)$ is the initial transmission of the sample, $N$ is the number density of chromophores, and $l$ is the sample thickness. The areas are often approximated by the product of the hight and width of the hole and the absorption band. ${ }^{18}$ Hence the hole area is in fact only the area under the zero-phonon hole, whereas the area under the inhomogeneous band contains all phonon contributions. For an optically thin sample the last term in eq 45 reduces to $1 / F_{B} A_{0}\left(\omega_{B}\right)$. Evaluation of the areas within the cw model of hole burning then yields

$$
\begin{gathered}
\int A_{0}(\omega) \mathrm{d} \omega={ }^{1} /{ }_{3} \sigma_{a c}^{0} \\
\int H_{\|}(\omega) \mathrm{d} \omega={ }^{3} /{ }_{5} f_{a b} f_{a c} \Phi F_{B} \sigma_{a b}^{0} A_{0}\left(\omega_{B}\right) t \\
\Phi_{B}=\frac{9 f_{a b} f_{a c} \sigma_{a b}^{0}}{5 \sigma_{a c}^{0}} \Phi
\end{gathered}
$$

For a resonant hole $(b=c)$ with large Debye-Waller factor the quantum yield of hole formation $\Phi_{B}$ can be larger than the photochemical quantum yield $\Phi$ since three-fifths of the anisotropically burnt molecules contribute to $H_{\|}$, whereas only onethird of the isotropically oriented molecules contribute to the inhomogeneous absorption band.

From cw hole-burning experiment on TPP in PMMA the quantum efficiencies for hole formation $\Phi_{B}=9 \times 10^{-4}$ at $T=$ $10 \mathrm{~K}^{17}$ and $\Phi_{B}=2 \times 10^{-3}$ at $T=20 \mathrm{~K}^{18}$ have been reported. With $f=0.54$ this corresponds to the photochemical quantum yield $\Phi$ $=1.7 \times 10^{-3}$ at $T=10 \mathrm{~K}$, which is somewhat lower than our value $\Phi=6 \times 10^{-3}$. The difference may be due to the error in determining the absolute photon flux during hole burning but may also be caused by the different sample preparation techniques. The samples in refs 17 and 18 were prepared by solvent casting, whereas our samples were prepared by bulk polymerization and contain no solvent.

We feel that a fit of the complete saturation curve should yield more reliable parameters than a linear extrapolation of data at early burning times. For shallow holes the signal-to-noise ratio is usually small, and it is difficult to judge from these data whether the linear approximation is still applicable. That the same value for the photochemical quantum yield is obtained in hole burning with weak and strong light pulses (see Table 1) is an additional argument in favor of the fitting method. Furthermore, in the strong field case $\Phi$ and the excitation rate $k_{X}^{0}$ are independent parameters and the precise value of the fluence is not needed for the determination of $\Phi$.

4.4. Vibronic Satellite Holes. After hole burning into the origin band of an electronic transition vibronic satellite holes are often observed in the inhomogeneous vibronic absorption bands belonging to the same electronic transition. Vibronic satellite holes can also be observed in the electronic origin band after hole burning into the vibronic band. The latter method is favorable when the Franck-Condon factor for the origin is largest.

The shape of the satellite hole is given by the convolution of the distribution function of the vibrational frequencies within the electronic state with the hole shape for a fully correlated ensemble (see eq 13). The distribution function may be approximated by a Gaussian with width $B_{G}$. The shape of the zero-phonon hole for the fully correlated ensemble is a Lorentzian with width $B_{L}$ $=2 \Gamma_{a b}+2 \Gamma_{a c}$. The convolution of a Gaussian with a Lorentzian produces the voigt profile. ${ }^{19}$ Its width $B_{V}$ is a function of $B_{G}$ and $B_{L}$, and both parameters can be obtained from a fit if they are of comparable size.

The line profile of a satellite hole burnt at $\bar{\nu}_{B}=16524 \mathrm{~cm}^{-1}$ and observed with peak at $15569 \mathrm{~cm}^{-1}$ (corresponding to a vibrational wavenumber of $955 \mathrm{~cm}^{-1}$ ) is shown in Figure 5A together with the fit of a voigt profile for the zero-phonon line and a Gaussian for the phonon sideband. The fit yields $B_{G}=3.1$ $\mathrm{cm}^{-1}$ and $B_{L}=1.3 \mathrm{~cm}^{-1}$. With $2 \Gamma_{a c}=0.24 \mathrm{~cm}^{-1}$ measured for the resonant hole in the electronic origin a homogeneous width $2 \Gamma_{a b}=1.1 \mathrm{~cm}^{-1}$ is obtained for the vibronic transition. From the width of the resonant hole in the inhomogeneous vibronic band a somewhat larger value $2 \Gamma_{a b}=1.4 \mathrm{~cm}^{-1}$ is obtained. There are, however, several vibrational subensembles resonant with the laser at $\bar{\nu}_{B}=16524 \mathrm{~cm}^{-1}$, and all contribute to the resonant hole.

Hole burning with larger fluence produces the satellite holes shown in Figure 5B. All spectra refer to the same baseline. The maximum relative hole depth converges to a value between 0.5 and 0.6 , consistent with a Debye-Waller factor of 0.54 . The strong background can be explained by the congestion of holes produced by other vibronic subensembles excited into their phonon sidebands. To correct for the strong background the relative hole depth is obtained as the amplitude of the voigt profile fitted to the central part of the zero-phonon hole divided by the initial optical density. The saturation of these relative hole depths with increasing number of laser shots is shown for the satellite hole at $955 \mathrm{~cm}^{-1}$ in Figure 6. The behavior is well described for both polarizations by the model of $\mathrm{cw}$ hole burning, i.e., the parameters 


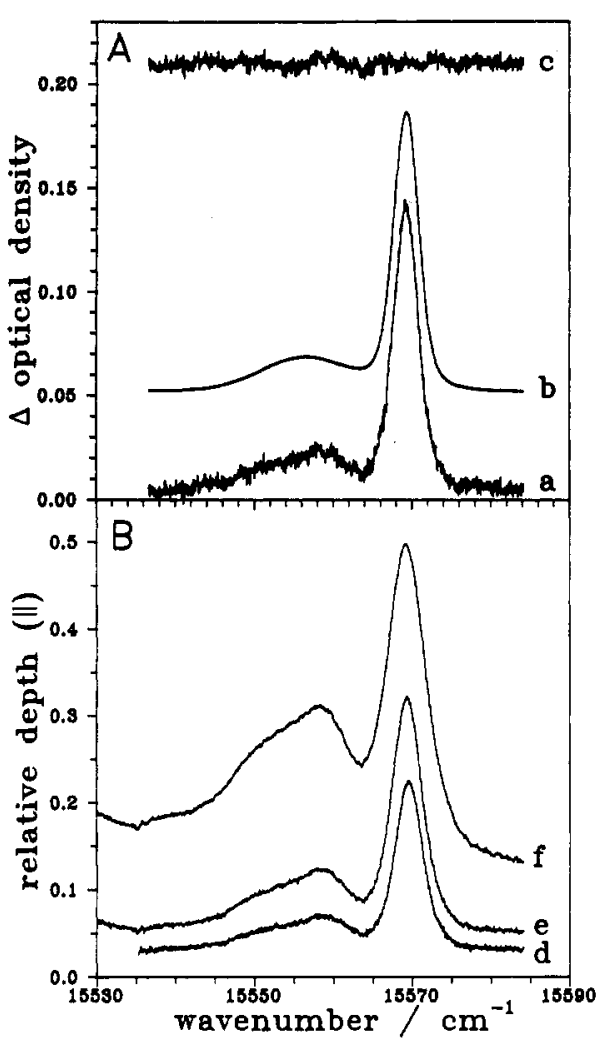

Figure 5. (A) (a) Profile of a vibronic satellite hole $\left(\Delta \bar{\nu}=955 \mathrm{~cm}^{-1}\right)$ burnt by 970 laser pulses with fluence of ca. $I \tau \approx 40 \mu \mathrm{J} \mathrm{cm}-2 /$ pulse into a vibronic absorption band of TPP in PMMA at $T=10 \mathrm{~K}$. (b) Fit of a Voigt function $\left(B_{L}=1.21 \mathrm{~cm}^{-1}, B_{G}=3.09 \mathrm{~cm}^{-1}\right)$ to the zero-phonon line and a Gaussian $\left(B_{G}=13 \mathrm{~cm}^{-1}\right)$ to the phonon sideband. (c) Residuals of the fit. (B) Hole profiles after 3570 (d), 7300 (e), and 45700 (f) laser pulses.

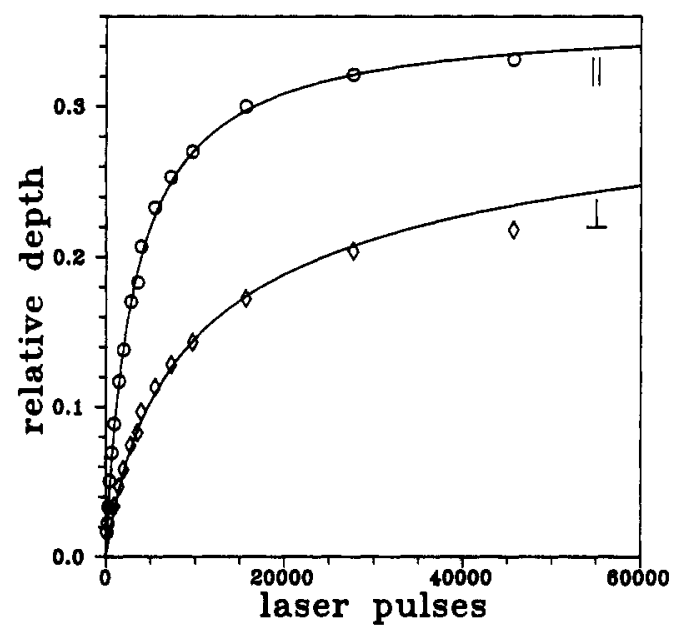

Figure 6. Saturation of the relative hole depth $H_{\|}$and $H_{\perp}$ of the vibronic satellite hole $\left(\Delta \bar{\nu}=955 \mathrm{~cm}^{-1}\right)$ of TPP in PMMA at $T=10 \mathrm{~K}$. One laser pulse corresponds to a fluence of $I \tau \approx 40 \mu \mathrm{J} \mathrm{cm}^{-2}$. Upper curve: fit of eq 28 to the data for $H_{\text {with }} H_{\text {sat }}=0.36$ and $Y_{\tau}=\Phi f_{a b} \sigma_{a b}^{0} F_{B} \tau / \Gamma_{a b}=$ $3.6 \times 10^{-3}$. Lower curve: simulation with the same parameters for $H_{\perp}$.

obtained from the fit to $H_{\|}$produce an equally good simulation for $H_{\perp}$. For infinite burning time both curves approach a limiting hole depth $H_{\text {sat }}=0.36$, which is smaller than the Debye-Waller factor obtained for the resonant hole in the electronic origin.

Within the cw model of hole burning the plot of $\rho$ vs $H_{\|}$depends only on the angle $\delta$ between the transition moments and the limiting hole depth $H_{\text {sat }}$. Figure 7 shows simulated curves for several values of $\delta$ with $H_{\text {sat }}=0.36$. It is known that the vibrational mode at $955 \mathrm{~cm}^{-1}$ is totally symmetric and that the corresponding vibronic transition is polarized parallel to the electronic origin. 9 The experimental data for the satellite hole (circles) are in good

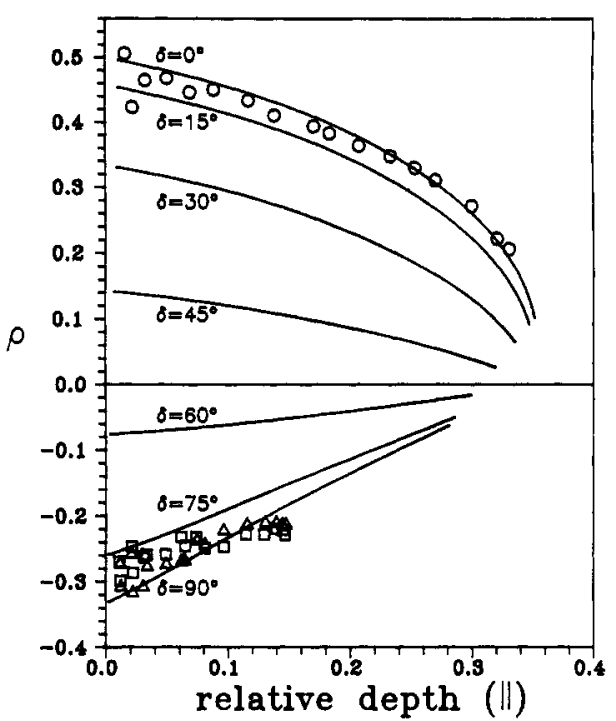

Figure 7. Saturation of the degree of polarization $\rho$ of spectral satellite holes with increasing hole depth, simulated for $H_{\text {sat }}=0.36$ and several angles between the transition dipoles. Experimental data are shown for three satellite holes of TPP in PMMA corresponding to vibrational wavenumbers of $955 \mathrm{~cm}^{-1}$ (circles), $1194 \mathrm{~cm}^{-1}$ (squares), and $1207 \mathrm{~cm}^{-1}$ (triangles).

agreement with the expectation for $\delta=0^{\circ}$. Two other modes at vibrational wavenumbers of 1194 and $1207 \mathrm{~cm}^{-1}$ belong to vibronic transitions polarized perpendicular to the electronic origin. ${ }^{9}$ Experimental data for the degree of polarization of the corresponding satellite holes, burnt with increasing fluence at 16771 $\mathrm{cm}^{-1}$ and observed at 15577 and $15564 \mathrm{~cm}^{-1}$, are also shown in Figure 7 (triangles and squares). For small hole depth the data fall between the theoretical lines for $\delta=75^{\circ}$ and the limiting value $\delta=90^{\circ}$. Hence it appears that this method can yield the angle between transition moments with an accuracy of ca. $15^{\circ}$.

\section{Conclusions}

We have presented a theoretical description of saturation effects in line shapes and anisotropy of resonant and vibronic satellite spectral holes. Our model includes a possible inhomogeneity in the distribution of vibrational frequencies in the excited state and saturation of the molecular system by the light pulses used for hole burning. The resulting formulas require a two-dimensional integration over one frequency and one orientational coordinate. In the limit of broad inhomogeneous distributions and small hole depths burnt with low light intensity, these integrals can be performed analytically. For other cases numerical integration routines were used. We are presently developing efficient recursive algorithms for the calculation of these integrals.

Our model calculations, though seemingly already complex, still contain two simplifications which are mainly responsible for the deviations from the experiment especially for large hole depths. The first simplification is the neglect of hole burning through excitation into phonon sidebands. The second is the restriction to only one resonant transition of the chromophores during hole burning. There is no principal difficulty in including the complete form of the cross section (eq 21) or a sum over several resonant states into the expression for $\Delta N_{a}$, i.e., eq 28 in the $\mathrm{cw}$ model or eq 41 in the pulsed model. However, this will increase the number of adjustable parameters unless parameters for the phonon sidebands are accessible from other sources. An alternative and in our view more promising strategy aims at avoiding the complications caused by the phonon sidebands by experimental means. This can be done by lowering the temperature but also by the choice of matrix materials with less electron-phonon coupling. This interaction should be small in matrices made of frozen rare gases due to the absence of internal bond vibrations 
in these materials. Our first investigation of a photochemical reaction in solid rare gases produced indeed highly resolved vibronic satellite hole spectra with no detectable contamination by phonon sidebands.

In the case of hole burning with strong light pulses that saturate the resonant subensemble of chromophores a simultaneous fit of the model equations to $H_{\|}$and $\rho$ yields the photochemical quantum yield $\Phi$ and the excitation rate $k_{X}^{0}$ as independent parameters. Hence for the determination of $\Phi$ a precise knowledge of the absolute laser intensity at the sample is not needed.

Acknowledgments. The authors gratefully acknowledge generous support by Prof. F. P. Schäfer (Ableilung für Laserphysik, Max-Planck-Institut für biophysikalische Chemie in Göttingen) where the experimental work has been performed. Part of the apparatus was financed by the Deutsche Forschungsgemeinschaft through the Leibniz Prize awarded to Prof. Schäfer and through project SFB 93/D8 (Photochemie mit Lasern). Support was also given by the Fonds der Chemischen Ind.

\section{References and Notes}

(1) Perrin, F. Ann. Phys. 1929, 12, 169.
(2) Osad'ko, I. S.; Soldatov, S. L.; Jalmukhabetov, A. U. Chem. Phys. Lett. 1985, 118, 97 .

(3) Köhler, W.; Breinl, W.; Friedrich, J. J. Phys. Chem. 1985, 89, 2473.

(4) Kador, L.; Schulte, G.; Haarer, D. J. Phys. Chem. 1986, 90, 1264.

(5) de Vries, H.; Wiersma, D. A. J. Chem. Phys. 1980, 72, 1851.

(6) Friedrich. J.; Haarer, D. J. Chem. Phys. 1983, 79, 1612

(7) Nickel, B. J. Lumin. 1989, 44, 1.

(8) Hearn, A. C.; REDUCE version 3.4, Report CP 78, RAND, 1991, Santa Monica, CA 90407-2138.

(9) Dick, B. Chem. Phys. 1989, 136, 429.

(10) Zeller, R. C.; Pohl, R. O. Phys. Rev. 1971, B4, 2029. 103 .

(11) Tamkivi, R.; Renge, I.; Avarmaa, A. Chem. Phys. Lett. 1983, 103,

(12) Gradyushko, A. T.; Tsvirko, M. P. Opt. Spectrosc. 1971, 31, 291.

(13) Dzhagarov, B. M.; Sagun, Ye. I.; Bondarev, S. L.; Gurinovich, G. P. Biofizika 1977, 22, 565.

(14) Saikan, S.; Imaoka, A.; Kanematsu, Y.; Sakoda, K.; Kominami, K.; Iwamoto, M. Phys. Rev. 1990, B41, 3185.

(15) Moerner, W. E.; Gehrtz, M.; Huston, A. L. J. Phys. Chem. 1984, 88, 6459.

(16) Furusawa, A.; Horie, K.; Kuroki, K.; Mita, I. J. Appl. Phys. 1989, 66,6041 .

(17) Furusawa, A.; Horie, K. J. Chem. Phys. 1991, 94, 80.

(18) Horie, K.; Ikemoto, M.; Suzuki, T.; Machida, S.; Yamashita, T.; Murase, N. Chem. Phys. Lett. 1992, 195, 563.

(19) The Voigt profile is the imaginary part of the complex error function. For a detailed description and numerical approximations see: Gautschi, W. Error Function and Fresnel Integrals in Abramowitz, M.; Stegun, I. Handbook of mathematical functions; Dover: New York, 1965; pp 294ff. 